\title{
Efectividad de la aplicación de un Programa de Educación para la Salud en Preescolares
}

Effectiveness of the implementation of a program of Health Education in Preschool
Eficácia da implementação de um programa de Educação em Saúde no pré-escolar
Fecha de Recepción

16 de diciembre de 2015

Beatriz Juana Cardozo

Profesora Titular - Cátedra Práctica Clínica

Preventiva II.

Te. 03794.70050I.

E-mail: malusacardozo2@hotmail.com

Silvia Rita Pérez

Prof. Adjunta - Cátedra Práctica Clínica Preventiva

II.

Patricia Alejandra Vaculik Auxiliar Docente - Cátedra Práctica Clínica

Preventiva II.

Elena Griselda Sanz

Auxiliar Docente - Cátedra Práctica Clínica

Preventiva I.

\section{Aceptado para su publicación}

3 de abril de 2016

\section{Resumen}

Introducción: La salud bucal es un componente importante de la salud general, así como también resulta fundamental para el adecuado desarrollo del individuo. El objetivo fue comprobar la efectividad del Programa de Promoción y Educación para la Salud en niños de edad pre-escolar en el Jardín Pinocho de la ciudad de Corrientes durante el año 2013. Materiales y métodos: se realizó un estudio descriptivo, de corte longitudinal, en II4 niños entre 3 a 5 años. Se les realizó examen clínico y posterior registro en la Historia Médica-Dental, de la frecuencia de cepillado dental y momentos de azúcar inicial y posterior a la aplicación del Programa. Resultados: El 63,15\% de niños aumentaron a 4 veces el cepillado diario. Disminuyo a 3 veces por día de consumo de alimentos azucarados. Conclusiones: La aplicación del Programa ha evidenciado cambios importantes en la adquisición de nuevos hábitos de higiene bucal y consumo de azúcar.

\section{Palabras claves}

Preescolares, salud bucal, programa, educación.

\section{Summary}

Introduction: Oral health is an important component of overall health, and is also essential for the proper development of the individual. The objective was to test the effectiveness of the Program for Promotion and Health Education 
in children pre-school age in the Garden City Pinocchio Corrientes during 2013. Materials and Methods: A descriptive study was conducted slitting, I I 4 children between 3-5 years. He underwent clinical examination and subsequent registration in the Medical-Dental, History of the frequency of tooth brushing and time of initial and subsequent to the implementation of sugar. Results: $63.15 \%$ of children increased to 4 times daily brushing. Decreased to 3 times a day of consumption of sugary foods. Conclusions: The implementation of the program has shown significant changes in the acquisition of new habits of oral hygiene and sugar intake.

\section{Keywords}

Preschool, oral health program, education.

\section{Resumo}

Introdução: saúde oral é um componente importante de saúde geral, e também é essencial para o bom desenvolvimento do indivíduo. $O$ objetivo foi testar a eficácia do Programa de Promoção e Educação para a Saúde em crianças de idade pré-escolar no Garden City Pinocchio Corrientes durante 2013. Materiais e Métodos: Estudo descritivo de corte foi realizado, I I 4 crianças entre 3-5 anos. Ele foi submetido a exame clínico e posterior registo na Médico-Odontológica, História da frequência de escovação dos dentes e hora de inicial e subsequente à implementação de açúcar. Resultados: 63,15\% das crianças aumentou para 4 vezes escovação diária. Diminuiu para 3 vezes por dia de consumo de alimentos açucarados. Conclusões: $A$ execução do programa tem mostrado mudanças significativas na aquisição de novos hábitos de higiene oral e ingestão de açúcar.

\section{Palavras chave}

Pré-escolar, programa de saúde bucal, educação.

\section{Introducción}

La salud bucal es un componente importante de la salud general, así como también resulta fundamental para el adecuado desarrollo del individuo, como parte integral del complejo craneofacial, que participa en funciones vitales, entre ellas la alimentación, la comunicación, el afecto y la autoestima'.

Uno de los principales problemas de salud pública, a nivel mundial, dada su alta prevalencia, son las enfermedades bucodentales. Según estudios recientes, la caries dental es la enfermedad que padece aproximadamente $90 \%$ de la población en América Latina; lo más alarmante es que comienza en la etapa temprana de la vida y se incrementa a medida que el individuo crece $^{2}$.

La educación en salud como componente básico de la promoción de salud, propicia cambios de conceptos, comportamientos y actitudes frente a la salud oral y a la enfermedad; refuerza conductas positivas a nivel colectivo e individual y reconoce la necesidad particular de planificar y definir estrategias aplicables a su estilo de vida!.

El establecimiento de programas preventivo promocionales en escuelas en niños y adolescentes ha sido evaluado mostrando resultados alentadores en la mejora de conocimientos, desarrollo de buenos hábitos de higiene y disminución en los niveles de placa y salud gingival, proponiendo que la caries $y$ enfermedad periodontal pueden ser controladas por un cepillado regular con una pasta fluorada ${ }^{3}$.

La prevención en salud oral son aquellas actividades que buscan controlar los factores de riesgo causantes de las diferentes patologías orales. Tiene un elemento común, que es el control de la placa bacteriana, mediante una adecuada higiene oral, teniendo en cuenta que la supresión de la actividad microbiana ha demostrado ser eficaz en la prevención del desarrollo de lesiones cariosas y pérdida de inserción periodontal'.

La promoción del autocuidado se proclama frecuentemente como la estrategia más importante en la salud primaria dental. El cepillado dental ha sido, por muchos años, el principal mensaje de educación dado a niños, adolescentes y adultos, se basa la supuesta ayuda a eliminar la placa y facilita el contacto íntimo del fluoruro contenido en la pasta dental con los dientes. Para promover eficazmente el cepillado dental es necesario entender los factores asociados con esta práctica/conducta de higiene ${ }^{4}$.

En la formación de hábitos adecuados de higiene oral, la promoción de la salud oral y la prevención de la enfermedad juegan un papel 
preponderante y se consideran acciones fundamentales para el control de la placa bacteriana'.

Debido a que la prevalencia de caries dental esta asociada a una baja frecuencia de cepillado y elevado consumo de golosinas se sugiere que los principales objetivos de los programas de salud dental deben estar dirigidos a lograr el cepillado dental diario, reducir el consumo de golosinas e incrementar los conocimientos sobre salud oral; siendo que principalmente estos programas deben estar dirigidos a población rural y de menor nivel sociocultural ${ }^{5}$.

\section{Materiales y métodos}

Se realizó un estudio descriptivo, de corte longitudinal, en I I 4 niños entre 3 a 5 años que concurrieron al Jardín de Infantes $N^{\circ} 7$ Pinocho de la Ciudad de Corrientes durante el año 2013.

El objetivo del estudio fue comprobar la efectividad del Programa de Promoción y Educación para la Salud en niños de edad pre-escolar en el Jardín Pinocho de la ciudad de Corrientes durante el año 2013.

Este estudio se llevo a cabo en el jardín de infantes durante el horario de actividades, a todos los niños se les realizó un examen clínico y posterior registro en la Historia Médica-Dental previa firma del consentimiento Informado de los tutores. (Autorizado por el Comité de Ética de la Facultad de Odontología de la UNNE. Dictamen $N^{\circ} 43 / / 2$ ). En la historia clínica se registraron la frecuencia de cepillado dental y los momentos de azúcar inicial, posteriormente se aplicó el Programa de Educación para la Salud, y al finalizar el mismo se registraron nuevamente la frecuencia de cepillado dental y los momentos de azúcar. El estudio se realizó en dos etapas: en la primera etapa se solicitó el consentimiento de la Comunidad Educativa, se calibró al personal investigador y luego se realizó el diagnóstico inicial. En la segunda etapa se llevó a cabo la Implemtación del Programa por Una Sonrisa Sana y Feliz, en el cual se brindaron charlas educativas acompañadas de material didáctico, con el fin de formar a los docentes, padres y alumnos en agentes multiplicadores, respecto a la Salud Bucal concientizándolos acerca de un cambio de actitud respecto a la frecuencia del cepillado dental, momentos de consumo de azúcar, así como también la necesidad de una dieta equilibrada. Posteriormente se realizó la organización, clasificación, tabulación, análisis y evaluación de los datos obtenidos. Elaboración de los resultados y conclusiones.

\section{Resultados}

Los datos fueron sometidos a un análisis estadístico. Se calcularon las medidas de tendencia central moda, media artimética y mediana. Los resultados obtenidos se presentaron en tablas y gráficos.

\section{Situación inicial}

Analizando los datos obtenidos con respecto a la frecuencia del cepillado dental en preescolares, el valor que más veces se repite dentro del conjunto de datos se denomina moda, en éste estudio presentó un valor de 2 , que nos indicó que la mayor cantidad de niños se cepilla los dientes 2 veces por día.

La Media es 2.25 que nos indica el promedio de los datos, es decir, nos informa el valor que obtendría cada uno de los individuos si se distribuyeran los valores en partes iguales, nos reveló que el promedio de veces que se cepillaron los dientes era de 2 veces por día.

La Mediana es 2, ésta medida nos permite conocer el valor que se encuentra exactamente en la mitad del conjunto de datos, determinando que la mitad de los datos se encuentran por debajo de este valor y la otra mitad por encima del mismo. Tabla $\mathrm{N}^{\circ} \mathrm{I}$.

El porcentaje de niños que se cepillan los dientes I vez al día fue de $25,4 \%$, 2 veces $35 \%$, 3 veces al día $27,1 \%$ y 4 veces al día $12,2 \%$. Gráfico $\mathrm{N}^{\circ} \mathrm{I}$.

Con respecto a los momentos del día que consumían alimentos azucarados el valor que más veces se repite dentro del conjunto de datos se denomina moda, con un valor de 6 que nos indicó que la mayor cantidad de niños presentó 6 momentos de azúcar diarios, siendo éstos valores no compatibles con salud. La Media es 3.96. La Mediana es 4, ésta medida nos permite conocer el valor que se encuentra exactamente en la mitad del conjunto de datos, determinando que la mitad de los datos se 
Tabla $N^{\circ}$ I. Frecuencia cepillado dental en preescolares antes de aplicar el Programa.

\begin{tabular}{clll} 
Frecuencia cepillado dental & FA & FAA & FR \\
\hline I & 29 & 29 & $25,4 \%$ \\
\hline $\mathbf{2}$ & 40 & 69 & $35 \%$ \\
\hline 3 & 31 & 100 & $27,1 \%$ \\
\hline 4 & 14 & 114 & $12,2 \%$ \\
\hline
\end{tabular}

Tabla $N^{\circ}$ 2. Momentos de azúcar en preescolares antes de aplicar el Programa.

\begin{tabular}{cllll} 
Momentos de azúcar & FA & FAA & FR \\
\hline I & 5 & 5 & $4,38 \%$ \\
\hline $\mathbf{2}$ & II & 16 & $7,63 \%$ \\
\hline $\mathbf{3}$ & 24 & 40 & $16,66 \%$ \\
\hline $\mathbf{4}$ & 30 & 70 & $26,31 \%$ \\
\hline $\mathbf{5}$ & 10 & 80 & $8,77 \%$ \\
\hline $\mathbf{6}$ & 34 & 114 & $28,82 \%$ \\
\hline
\end{tabular}

Tabla $N^{\circ} 3$. Frecuencia cepillado dental en preescolares posterior a aplicación del Programa.

\begin{tabular}{clll} 
Frecuencia cepillado dental & FA & FAA & FR \\
\hline I & 9 & 9 & $7,8 \%$ \\
\hline $\mathbf{2}$ & 12 & 21 & $10,5 \%$ \\
\hline 3 & 21 & 42 & $18,42 \%$ \\
\hline 4 & 72 & 114 & $63,1 \%$ \\
\hline
\end{tabular}

Tabla $N^{\circ} 4$. Momentos de azúcar en preescolares posterior a aplicación del Programa.

\begin{tabular}{cllll} 
Momentos de azúcar & FA & FAA & FR \\
\hline I & 8 & 8 & $7,01 \%$ \\
\hline $\mathbf{2}$ & 21 & 29 & $18,42 \%$ \\
\hline $\mathbf{3}$ & 57 & 86 & $50 \%$ \\
\hline $\mathbf{4}$ & 11 & 97 & $9,64 \%$ \\
\hline $\mathbf{5}$ & 13 & 110 & $11,40 \%$ \\
\hline $\mathbf{6}$ & 4 & 114 & $3,50 \%$ \\
\hline
\end{tabular}

encuentran por debajo de este valor y la otra mitad por encima del mismo. Tabla $\mathrm{N}^{\circ} 2$.

Con respecto a los momentos del día que consumían alimentos azucarados, se observo que el $16,66 \%$ presentaron 3 momentos de consumo de azúcar, el 7,63\% presentaron 2 momentos de azúcar, el 4,38 \% presentaron I momento, siendo esto valores compatibles con salud. El $26,31 \%$ presentaron 4 momentos de azúcar, el 8,77\% 5 momentos y el 29,82 presentaron 6 momentos de azúcar, siendo esto valores no compatibles con salud. Gráfico N 2 .

\section{Luego de la aplicación del Programa}

Analizando los datos posterior a la Aplicación del programa observamos con respecto a la frecuencia del cepillado dental en preescolares, el 
Gráfico $N^{\circ}$ I. Relación del número de preescolares con la Frecuencia de cepillado dental antes de aplicar el Programa.

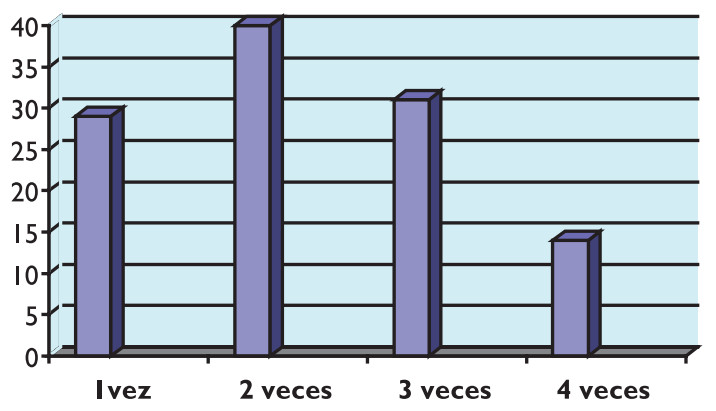

valor que más veces se repitió dentro del conjunto de datos, la moda, fue 4 que nos indicó que la mayor cantidad de niños se cepilla 4 veces por día los dientes. Aquí podemos evidenciar como luego de que los niños recibieran las medidas educativas en salud bucal han mejorado incrementando de 2 veces por día que se cepillaban antes de aplicar el Programa a 4 veces luego de la aplicación del mismo.

La Media es 3,75 que nos indico que el promedio de veces que se cepillaron los niños los dientes fue de 4 veces por día. Con éstos valores podemos observar como luego de la implementación del Programa, han mejorado incrementando el número de veces que se cepillaban por día que era 2 a 4 veces por día luego de la aplicación del mismo. La Mediana tiene un valor de 4. Tabla $\mathrm{N}^{\circ} 3$.

El porcentaje de niños que se cepillan los dientes 4 veces al día ha aumentado a 63,15\%, 3 veces al día $18,42 \%$, 2 veces $10,5 \%$ y I vez al día el 7,8\%. Con éstos resultados podemos observar como luego de la implementación del Programa, ha aumentado el porcentaje de preescolares que se cepillan los dientes 4 y 3 veces por día. Gráfico $\mathrm{N}^{\circ} 3$.

Con respecto a los momentos del día que consumían alimentos azucarados, se observo que el valor de la moda, fue 3 que nos indicó que la mayor cantidad de niños consumió alimentos azucarados 3 veces por día, donde podemos observar como luego de que los niños recibieran las medidas educativas respecto de dieta, alimentos cariogénicos y no cariogénicos
Gráfico $\mathbf{N}^{\circ}$ 2. Relación del número de preescolares con los momentos azúcar antes aplicar programa.

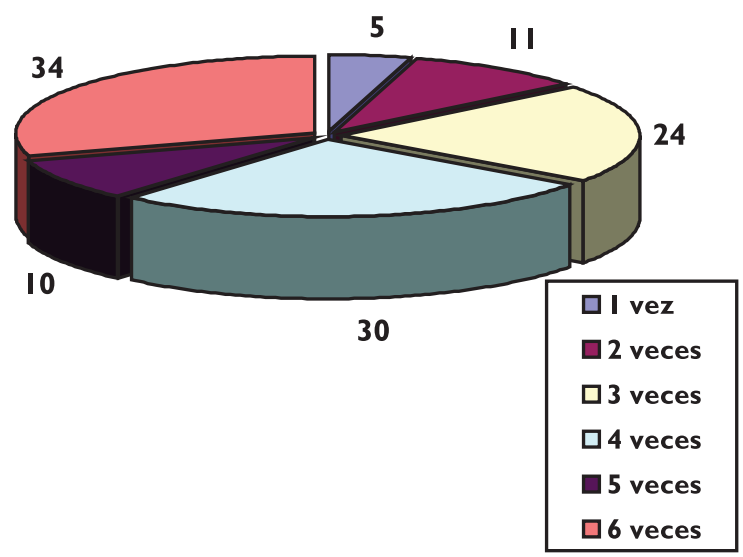

han mejorado disminuyendo de 6 veces por día, que consumían alimentos azucarados antes de aplicar el Programa a 3 veces luego de la aplicación del mismo, siendo esto valores compatibles con salud.

La Media es 3,09 que nos indico que el promedio de veces que consumieron alimentos azucarados fue de 4 veces por día. Con éstos valores podemos observar como luego de la implementación del Programa, han mejorado disminuyendo el número de momentos de azúcar a 3 veces por día, siendo esto valores compatibles con salud. La Mediana tiene un valor de 3. Tabla $\mathrm{N}^{\circ} 4$.

Con respecto a los momentos del día que consumían alimentos azucarados, se observo que el $7,01 \%$ de los niños presentaron I momento de consumo de azúcar, el $18,42 \%$ presentaron 2 momentos de azúcar, el $50 \%$ presentaron 3 momentos, siendo estos valores compatibles con salud, mientras que el $9,64 \%$ los niños presentaron 4 momentos de azúcar, el II,40\% 5 momentos y el 3,50\% presentaron 6 momentos de azúcar. Con éstos resultados podemos observar como luego de la implementación del Progra$\mathrm{ma}$, ha aumentado el porcentaje de preescolares que presentaron menor número de momentos de azúcar diarios. Gráfico $N^{\circ} 4$.

\section{Discusión}

El niño en edad preescolar se desenvuelve en un ambiente sociocultural (escuela-hogar) donde juega un papel importante las conductas que se adquieren desde temprana edad, que de 
Gráfico $N^{\circ} 3$. Relación del número de preescolares con la Frecuencia de cepillado dental posterior a la aplicación el Programa.

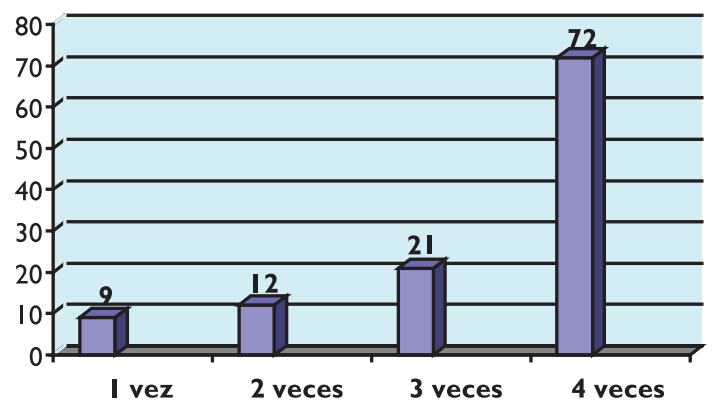

acuerdo a Laurell (1992) son predictores y determinantes de los factores de riesgo ${ }^{6}$. Motivo por el cual es primordial que en el hogar del niño, se apliquen conductas saludables, para que desde tempranamente vaya adquiriendo hábitos saludables de higiene bucal; esas conductas saludables deben ser reforzadas en el Jardín por parte de los maestras.

Los resultados obtenidos en éste estudio coinciden con los hallados por el Prof. Od. Cammarano Pellegrino, $\mathrm{F}$, en una investigación donde aplicaron un Programa Educativo Preventivo Odontológico a un total de 239 niños y niñas de la Unidad Educativa María auxiliadora; evidenciando los cambios y las modificaciones favorables en la conducta del cepillado dental posterior a la aplicación del mismo ${ }^{7}$.

Los hallazgos obtenidos en éste estudio coinciden con los referidos por la Mgter Sence-Campos, $R$, donde luego de la aplicación de medidas preventivas en una población de preescolares se logró incrementar el número de veces que se cepillaban los dientes diariamente $80,7 \%$, este mayor porcentaje también se puede atribuir a la mayor preocupación que muestran las madres por sus hijos más pequeños, y la mayor asistencia a las capacitaciones de padres que son realizadas también dentro del Programa Salud Bucal ${ }^{8}$.

Numerosos estudios han demostrado que el biofilmdental, es el responsable principal de las dos patologías más prevalentes en la cavidad bucal. Los resultados hallados en éste estudio coinciden con los obtenidos por Cardozo, B. y col. en alumnos de la Escuela Familia Agrícola
Gráfico $\mathbf{N}^{\circ} 4$. Relación de cantidad de preescolares con los momentos azúcar posterior aplicar programa.

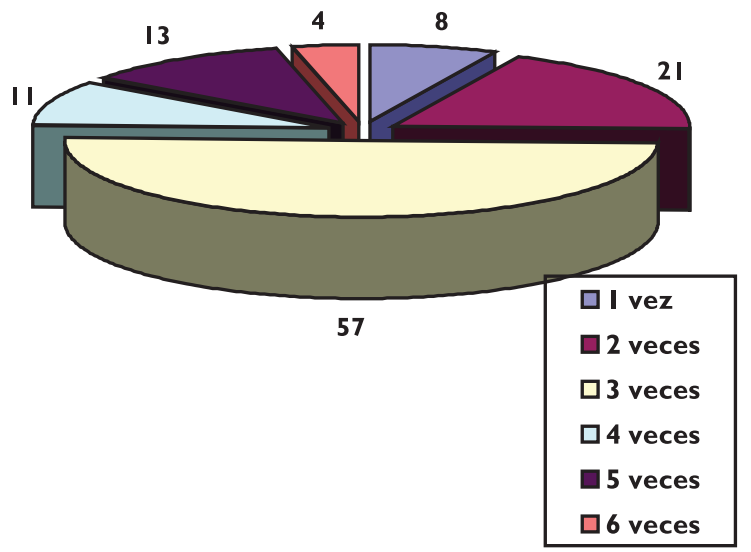

en San Miguel, Corrientes donde se demuestra una alta prevalencia de caries de $97,2 \%$, donde el sexo masculino presenta el $55 \%$ con respecto al sexo femenino que presenta el $37,5 \%$ de caries dental'.

Otro estudio realizado por Fernandez, $\mathrm{S}^{48}$ y col demostró que el cepillado dental se asocia a la salud bucodental, siendo el efecto positivo del cepillado superior al de una dieta correcta para prevenir caries ${ }^{10}$.

\section{Conclusión}

Este estudio se realizó en niños preescolares con edades comprendidos entre los 3 y 5 años de edad de ambos sexos, que concurrieron al Jardín de Infantes $N^{\circ} 7$ Pinocho de la Ciudad de Corrientes durante el año 2013.

Se han analizado los hábitos de higiene bucal frecuencia del cepillado dental y momentos de consumo de azúcares anterior y posterior a la aplicación del programa.

En base a los resultados obtenidos se observó que la aplicación del Programa ha evidenciado cambios importantes en la adquisición de nuevos hábitos de higiene bucal y en el consumo de azúcar de los preescolares que participaron en él. Esto demuestra que hubo modificaciones favorables en la conducta del cepillado dental y dieta por parte de los niños, siendo esto sumamente importante para ellos, ya que la incorporación de hábitos saludables de higiene bucal y dieta contribuirá al buen estado bucodental. 


\section{Bibliografía}

I. UNICOC. Guía Práctica Clínica en Salud Oral. Bogotá D.C., 2010; 4.

2. Argentina, Ministerio de Salud, Secretaría de Ambiente y Desarrollo Sustentable; Programa de Naciones Unidas para el Medio Ambiente. Argentina 2006: Indicadores ambientales. Iniciativa latinoamericana y caribeña para el desarrollo sostenible. Indicadores de seguimiento. Buenos Aires; 2006.

3. Tai BJ, Jiang H, Du MQ, Peng B. Assessing the effectiveness of a school-based oral health promotion programme in Yichang City, China. Community Dent Oral Epidemiol 2009; 37: 391-8.

4. Medina SCE,Segovia VA,Rodriguez RE. Asociación del nivel socioeconómico con la higiene bucal en preescolares bajo el programa de odontología preventiva del IMSS en Campeche. Gac Méd Méx 2006; 142 (5): I34-45.

5. Smyth E, Caamaño $F$. Factors related to dental health in 12-year-old children : a cross-sectional study in pupils. Gac Sanit 2005; 19 (2): I I3-9.

6. Laurell,C. (1986): El estudio social del proceso salud-enfermedad en América Latina. Cuadernos Médico Sociales. Links.

7. Cammarano Pellegrino, F. EFICACIA DE UN PROGRAMA DE SALUD BUCAL DESARROLLADO POR PROMOTORES ESCOLARES. Acta odontológica venezolana. 2013; 51 (4) ISSN 000 I-6365.

8. Sánchez-Huamán, Y; Sence-Campos, R. Efectividad de un Programa Educativo preventivo para mejorar hábitos de higiene y condición de higiene oral en escolares. Kiru 20I2; 9 (I) ISSN 1812-7886

9. Cardozo B., González Meana, M., Tropeano, N. Estudio Epidemiológico Estado de Salud dental en alumnos. Escuela Familia Agrícola- San Miguel- Ctes. Revista Facultad de Odontología FOUNNE; 2006 16-18.

10. Fernández, S., Sánchez, A.,Quintanilla, J.,Mallón, S. Relevancia clínica del cepillado dental y su relación con la caries-Atención Primaria de la Salud. 2009 (42) 372-379.

II. Almagro Nievas D, Benítez Hita JA, García Aragón MA, López Lorca MT. Incremento del índice de dientes cariados, perdidos por caries y obturados, entre escolares de Loja, España, Salud Publica Méx. 2002; 43: 192-198.
12. Barrancos Mooney, J. Operatorias Dental Integración Clínica. $4^{\mathrm{a}}$ ed. Buenos Aires Argentina: Editorial Médica Panamericana; 2008.

13. Zaror Carlos; Pineda Patricia; Villegas Mónica. Estudio clínico del primer molsr permanente en niños de 6 años de edad de la comuna Calbuco, Chile. Acta Odontológica Venezolana. Volumen $49 \mathrm{~N}^{\circ} 3 / 2011$.

14. Cid María del Carmen, Martínez I, Morales JM. Ingestión de azúcares en niños menores de I año. Revista Médica Electrónica. 28 (I): I 13-6; 2008.

15. Rivera-Hermosillo G, Martínez-Torres J, Hernández-Laguna $E$. Caries dental e higiene bucal en adolescentes. Rev ADM 2006, LXIII (6): 23I234.

16. Battellino S; Cattoni, S; Escudero, M. et al. Medidas antropométricas, salud bucodental y desarrollo psicológico en preescolares de la Ciudad de Córdoba. Rev. Fac. Cienc. Méd. Córdoba, 32: 32I-42, 1974. [ Links ]

17. Yankilevich, E; Battellino, L. Prevalência de la caries dental en escolares de nivel primario de una región metropolitana de la provincia de Córdoba, Argentina. Rev. Saúde Pública, 26: 21023,1992. [ Links ]

18. Olmedo Sánchez A; Solorzano Villaseñor B; González Gaytan I; Díaz García B; Pérez Santana $R$. Prevalencia de caries en escolares de Pueblo nuevo, Santa Catarina Cuexcomatitlan, Mezquitic, Jalisco. Revista Odontológica Mexicana 2008; I2 (SI): SI0-S42. ISSN I870-199X.

19. García Crimi, G. Promoción de Salud Bucodental en establecimientos educativos del Valle de Uco de la Provincia de Mendoza. Facultad de Odontología UNCuyo. 20I0. Volumen 4. $\mathrm{N}^{\circ} \mathrm{I}$.

20. Evans, J. Sonríe Latinoamérica. Panamá. 200I.

21. Diaz Del Mazo, I. Sonrisas Saludables para capacitar adolescentes sobre Salud Bucal. Cuba. Medisan; 2003.

22. Elias, M. Programa Clínicas del Bebè. Perú. 200 I. 